\title{
Tissue responses to ischemia: local and remote responses for preserving perfusion of ischemic muscle
}

\author{
Jeffrey M. Isner \\ Tufts University School of Medicine and the Department of Medicine (Vascular Medicine) and Biomedical Research, \\ St. Elizabeth's Medical Center, Boston, Massachusetts, USA
}

Address correspondence to: Jeffrey M. Isner, St. Elizabeth's Medical Center, 736 Cambridge Street, Boston, Massachusetts 02135, USA. Phone: (617) 789-2392; Fax: (617) 779-6362; E-mail: VeJeff@aol.com.

The sequence of biological events that permits an organism to maintain tissue viability in the face of acute or chronic ischemia constitutes a fundamental survival response. Among mammalian species, this response may be best exemplified by the Israeli mole-rat (1), a creature that lives only in Egypt, Israel, and Syria. What is fascinating about this animal is that its entire life is spent underground in subterranean burrows at decidedly low oxygen tensions; accordingly, its tissues have been shown to be highly vascularized, and the vascular density is associated with upregulated endogenous expression of VEGF.

Among supraterranean species confronted with tissue ischemia localized to cardiac or skeletal muscle, at least two options are available. The first is to reduce demand for tissue oxygenation. This may be accomplished by "biochemical splinting," in which metabolic functions are converted from primarily aerobic to predominantly anaerobic. In the extreme, reduced blood flow may be associated with literal splinting, that is impaired wall motion characteristic of hibernating myocardium (2). Alternatively, "behavioral splinting" may be employed. For cardiac muscle, this may be achieved by reduced activity and, if necessary, pharmacologic therapies that reduce myocardial wall stress. For skeletal muscle, pharmacologic interventions are so limited that reduced levels of activity constitute the typical response to claudication. These adjustments, however, are necessary only if natural reparative mechanisms have failed to address the problem of ischemia successfully by restoring blood flow to the affected muscle group. Our current notions concerning the means by which this is achieved, as well as the factors that may selectively obviate this adaptive response, are the subject of this Perspective.

Natural response involves cytokine and cellular elements

Nature's response to the development of profound muscle ischemia includes upregulation of angiogenic growth factors and mobilization of circulating cellular elements that together enable development of an accessory vasculature. These events, not surprisingly, recapitulate many aspects of embryonic circulatory development.
As in the Israeli mole-rat, VEGF appears to be a key regulatory cytokine orchestrating the response to postnatal ischemia in humans and more familiar experimental organisms. In a murine model of hindlimb ischemia (3), for example, we observed that excision of the iliac and femoral arteries was followed by reduced blood flow and evidence of tissue necrosis documented by histochemical staining. Within $2-4$ days, tissue immunostaining and Western blots of skeletal muscle harvested from the ischemic limb documented upregulation of VEGF protein. This lasted for 28-35 days. Similar findings have been reported in response to transient myocardial ischemia (4).

Further evidence for the central role of VEGF in the neovascularization of ischemic tissues comes from two experimental interventions in the murine hindlimb ischemia model. The first involved administration of recombinant platelet factor 4, which inhibits angiogenesis by disrupting VEGF receptor-mediated signal transduction and/or by disrupting the binding of VEGF to cell surface heparan sulfates. To assess more specifically the role of VEGF in modulating angiogenesis in this system, we administered a VEGF-neutralizing antibody to animals with hindlimb ischemia and confirmed that recovery of blood flow, capillary density, and proliferative activity measured by incorporation of BrdU were all significantly depressed. Similar attenuation of spontaneous angiogenesis has been demonstrated in freshly cut aortic rings cultured in a serum-free collagen gel and treated with a neutralizing VEGF antibody.

While such a time-course analysis of tissue expression is not feasible in human subjects, at least two groups have now studied patients following acute myocardial infarction and documented a similar rise and fall in VEGF expression $(5,6)$.

Mechanisms of VEGF induction during ischemia D'Arcangelo et al. (7) recently suggested that tissue acidosis independent of hypoxia may constitute an important stimulus to upregulated VEGF expression. While the mechanism for upregulated VEGF expression in response to acidosis remains enigmatic, it is clear that hypoxia-induced VEGF expression is mediated by the 
binding of the transcription factor hypoxia-inducible factor-1 (HIF-1) to a hypoxia response element in the $V E G F$ promoter (8). Endomyocardial biopsy specimens retrieved from patients undergoing coronary artery bypass surgery exhibit increased HIF-1 levels, associated with acute ischemia or early infarction (5).

Increase in mRNA stability constitutes a second important control point for the hypoxic induction of VEGF in different cell lines (9). Stabilization of VEGF mRNA by hypoxia is thought to be mediated by the binding of sequence-specific RNA-binding proteins to sequences in both the $3^{\prime}$ and $5^{\prime}$ untranslated regions of VEGF mRNA. These interactions occur under the stress of hypoxia and extend the intrinsically short halflife of VEGF mRNA about fourfold (10).

A third feature of VEGF that is critical for facilitating an efficient and sensitive response to hypoxia is the presence of an internal ribosome entry site that permits capindependent translation by ribosomal scanning of its mRNA (10). This may be particularly important because the $5^{\prime}$ untranslated region of the VEGF mRNA has several features that interfere with efficient ribosomal scanning, including its length, high $\mathrm{G}+\mathrm{C}$ content that permits secondary structure formation, and a short open reading frame bounded by in-frame initiation and termination codons. Importantly, Stein et al. (10) have shown that internal ribosome entry onto the VEGF mRNA is not diminished by the development of hypoxia.

In vivo and in vitro studies document VEGF synthesis in skeletal (3) and cardiac myocytes (11), as well as in vascular endothelial cells (ECs) under conditions of hypoxia (3). Circulating T cells (12) and monocytes (13) provide another source of VEGF as they infiltrate into the necrotic/ischemic milieu. This property of infiltrating $T$ cells was first described in the development of tumor neovasculature (14). The critical nature of this contribution has perhaps been best demonstrated in $\mathrm{T}$ cell-deficient nude mice that in response to hindlimb ischemia undergo necrotic autoamputation due to retarded angiogenesis $(12,15)$.

\section{VEGF signaling and neovascularization}

There is also good evidence to suggest that coordinated upregulation of VEGF receptor expression is important not only for enabling, but indeed for localizing neovascularization. VEGF receptors are typically expressed at exquisitely low levels under quiescent circumstances. With the onset of hypoxia, however, expression of VEGF receptor-2 (KDR) has been shown to increase up to 13-fold in skeletal (16) and cardiac (4) muscle. The consequent differential in upregulated VEGF expression by ischemic versus normal tissues may play a critical role in limiting neovascularization to those sites where augmented perfusion is required.

Previous reports by Murohara (17), Ziche (18), and others suggest that VEGF-induced angiogenesis involves upregulation of nitric oxide (NO) expression, mediated in large part by the Akt proto-oncoprotein. Studies of the arterial wall after endothelial denudation show that NO can feed back to repress VEGF expression in the regenerated endothelium (19). Whether NO plays a similar role in limiting VEGF expression after reconstitution of limb perfusion remains to be clarified.

Endogenous revascularization often has a distinctive appearance when visualized radiographically using iodinated contrast agents. Such angiograms typically disclose a "corkscrew" appearance, once alleged to be specific for so-called Buerger's disease, but now recognized to be a characteristic feature of collateral vessels in general. Why such collaterals are crooked remains uncertain, but it is possible that such vessels represent the fusion of multiple neovascular segments that are joined together under the influence of certain angiogenic growth factors such as VEGF (20). A second possibility is that developing vessels transiently deviate, before re-establishing a correct course in the direction of an ischemic stimulus.

In addition to these relatively large, angiographically visible collateral vessels, the neovasculature that develops in response to ischemia includes a large number of vessels smaller than $180-200 \mu \mathrm{m}$, the limit of resolution for conventional angiographic imaging. DNA labeling studies in pig and dog models of myocardial ischemia established that improvement in collateraldependent flow typically results from proliferation of vessels less than $200 \mu \mathrm{m}$ in diameter (21). Using synchrotron radiation microangiography, Takeshita et al. (22) recently confirmed that neovascularization following VEGF gene transfer predominantly involved vessels less than $180 \mu \mathrm{m}$ in diameter.

The maturity and durability of the vessels that form in response to de novo or therapeutic neovascularization are the subject of intensive inquiry. Most investigators currently view the full maturation of developing neovasculature as a process that borrows from embryonic paradigms, including evidence that a cascade of angiogenic growth factors is required to elaborate a normal vascular network. Gene targeting experiments, for example, have indicated that the Tie-2 ligand angiopoeitin-1 (Ang-1) plays a critical role in neovascular maturation. No evidence has yet been published indicating that Ang-1 is upregulated in response to tissue ischemia, and indeed, in vitro studies (23) have failed to show that Ang-1 (in contrast to its relative angiopoietin-2 [Ang-2]) is upregulated in response to hypoxia or VEGF. Conditions in which Ang1 is upregulated postnatally remain largely undefined.

There is a similar lack of evidence implicating upregulated tissue expression of FGF in response to ischemia (24). VEGF has been shown to upregulate EC expression of both PDGF-A and PDGF-B. Together with gene targeting data that have demonstrated an absence of pericytes in $P D G F-B^{-/-}$mice, these findings suggest that 
VEGF-induced EC secretion of PDGF may function to recruit smooth muscle cells, including pericytes, to facilitate maturation of the neovasculature.

The durability of neovasculature that develops in response to ischemia does not appear, however, to depend upon persistent upregulation of these soluble factors. Clinical experience with patients in whom collateral vessels form in response to occlusion of coronary or lower-extremity medium-sized arteries indicates that such collateral vessels persist indefinitely. This is in marked contrast to neovasculature that develops in association with wound healing, including the healing of wounds induced by myocardial laser revascularization. The latter is ultimately associated with vascular regression, once the wound has healed. In contrast, vessels that develop to provide nutrient blood flow persist as long as the need for such accessory flow exists; this postnatal observation is consistent with the embryonic paradigm that blood flow principally determines which, among the plethora of blood vessels that form during embryogenesis, will undergo apoptosis and which will survive to term (25).

An additional cellular component of the response to ischemia involves bone marrow-derived circulating EC precursors, termed endothelial progenitor cells (EPCs) (26). Experimental hindlimb ischemia in mice, for example, increases the frequency of EPC-enriched population in the circulation by more than $400 \%$ (27). EPC differentiation, assessed by the number of cultured EPCs among mononuclear blood cells under EC-specific conditions, was similarly increased. The mouse cornea micropocket assay has been used to investigate the impact of enhanced EPC mobilization induced by ischemia on neovascularization in animals recovering from hindlimb ischemia. Neovascularization of the normally avascular mouse cornea is enhanced in animals with hindlimb ischemia, compared with nonischemic, sham-operated controls. Furthermore, when we repeated this procedure using mice that had received bone marrow that was marked such that marrow-derived ECs would express $\beta$-galactosidase, we detected a statistically significant increase in reporter gene expression in corneas of mice with hindlimb ischemia, relative to a sham-operated group. EPCs may thus participate in repair following ischemic injury, in a process controlled by the bone marrow via circulating cytokines and soluble receptors and/or adhesive molecules. The identity of such mediators is unknown.

\section{Pathologic attenuation of endogenous neovascularization}

Animal studies performed in a variety of species suggest that endogenous neovascularization in response to ischemia may be impaired in certain physiological or pathological states. In old mice ( 2 years old) and old rabbits (5 years old), angiogenesis in response to hindlimb ischemia is markedly impaired (28). Reductions in per- fusion pressure, angiographically visible collaterals, hindlimb blood flow, and capillary density were associated with reduced levels of VEGF expression.

Certain aspects of this impaired response have now come to light. Activation of HIF-1 by hypoxia is primarily mediated by the stabilization of HIF- $1 \alpha$ protein (8). In vitro studies from our laboratory and others suggest that hypoxia-induced stabilization of HIF-1 $\alpha$ and/or HIF-1-DNA binding activity $(29,30)$ are impaired with aging. Hypoxia-induced expression of VEGF or of reporter constructs driven by the VEGF promoter is significantly diminished in smooth muscle cells from older rabbits, a difference that is attributable to HIF-1 binding, since HIF-1 protein levels and DNA binding activity are reduced in these cells (29). HIF-1 protein has been shown to be rapidly degraded by the ubiquitin-proteasome system under normoxic conditions and to be stabilized by hypoxia through redox-induced changes. It is also possible that aging diminishes the ability of HIF-1 to bind to the hypoxia response element within the VEGF promoter or to form active heterodimers. Such posttranslational loss of function has previously been described with aging for other proteins and transcription factors.

Consistent with the notion that endogenous neovascularization represents a combination of cytokine and cellular responses, however, age-related defects are not confined to ligand upregulation. Recently completed analyses of patients with critical limb ischemia show that mobilization of bone marrow-derived EPCs is significantly attenuated in older individuals after VEGF gene transfer (31).

Impairment of endogenous neovascularization has also been demonstrated in murine $\left(A p o E^{-/-}\right)(12)$ and rabbit (Watanabe) (32) models of hypercholesterolemia. As is seen in animal models of advanced age, VEGF expression in tissue sections retrieved from the ischemic limbs of these dyslipidemic animals was markedly reduced. The inference that loss of VEGF expression associated with hypercholesterolemia is - as in old age - ultimately attributable to defects in HIF-1 expression and/or binding has been confirmed in the Watanabe rabbit, in which impaired angiogenesis could be rescued by gene transfer of a HIF-1 $\alpha /$ VP16 naked DNA hybrid (33). While we have recently found that hypercholesterolemia augments the population of circulating EPCs (C. Kalka, unpublished data), homing and integration of EPCs into foci of neovascularization appears to be markedly impaired.

Diabetes constitutes a third condition that is associated with impaired angiogenesis. First demonstrated in a murine (nonobese diabetic [NOD]) model of diabetes (34), this finding that has been recently confirmed in studies of human coronary collateral development (35). Reconstitution of hindlimb perfusion in the NOD mouse by VEGF gene transfer (34) again implicates VEGF as the critical angiogenic growth factor responsible for endogenous neovascularization in the setting of diabetes. 
Heterogeneity of endogenous neovascularization Even in the absence of the specific pathologic phenotypes cited above, there is now good evidence, both in animals and in patients, to indicate that natural heterogeneity is a characteristic and important feature of endogenous neovascularization. In mice, for example, Rohan et al. (36) have documented a tenfold range of response in growth factor-stimulated angiogenesis among different strains of inbred mice. This extended to a differential sensitivity to angiogenesis inhibitors, with at least one strain demonstrating complete resistance to such therapy.

In human subjects, it has long been appreciated that certain patients - without a distinctive phenotype are "good collateral formers," while other patients are "poor collateral formers." Indeed, it is not at all uncommon to see patients with peripheral artery disease who, in spite of extensive lower extremity arterial occlusions, remain nearly asymptomatic because of a naturally robust collateral network. Past investigations of patients with coronary artery disease suggested that clinical prognosis may be determined by the extent of endogenous coronary collaterals supplying blood flow to the myocardial bed subtended by an occluded artery.

The possibility that this longstanding clinical notion has a genuine molecular basis is supported by work from Schultz et al. (37). These researchers evaluated the response of VEGF mRNA to hypoxia in monocytes harvested from patients undergoing coronary angiography and found that hypoxic induction of VEGF is significantly reduced in patients with poor collateral development. The significant difference in the induction of VEGF was maintained even after adjustment of data for variables such as age, diabetes, and hypercholesterolemia. Consistent with this clinical notion are the results of gene targeting experiments demonstrating deficient myocardial vascularity leading to an ischemic cardiomyopathy in mice lacking two of the three principal VEGF-1 isoforms (164 and 188) (38). Whether such impaired vascularization results from an overall reduction in VEGF expression or from differential expression of these isoforms remains to be clarified.

Individual variations in the potential for endogenous neovascularization are not likely to be limited to upstream dysregulation of HIF-1-mediated VEGF expression. Defective expression of tissue metalloproteinases, tissue plasminogen activators, or other components of the cascade responsible for neovascularization, including variations in intracellular signaling may prove to be contributory. Such variability between individual organisms is further complicated by what appears to be tissue-specific variation (39). For example, retinal hypoxia in diabetics induces VEGF upregulation, leading to pathologic neovascularization; these same patients may present with limb ischemia due to the above-described paucity of limb collaterals related to locally reduced expression of VEGF. Tissue-specific variations may also involve variable morphological features of the neovasculature and hence may be qualitative as well as quantitative (40).

Parenthetically, it is not yet clear that individual variations in the potential for endogenous neovascularization of ischemic tissues denote a similarly variable propensity for vascularizing neoplasms. The two mechanisms may well be quite independent. The source cells responsible for cytokine upregulation in response to tissue ischemia appear to be tightly regulated, allowing cytokine expression to be extinguished under certain circumstances. In contrast, the tumor cells that provide the cytokines seen in a neoplasia are, by definition, poorly regulated. Once switched on (41), they may boost the expression of angiogenic growth factors and promote uncontrolled vascular development.

\section{Future}

It is interesting to speculate that, as human lifespans increase, the role of angiogenic growth factor receptors and their cognate ligands may assume increased importance and exert novel evolutionary pressures on our species. When the average human lifespan was limited to 30 years, trauma and infection led to deaths well before an individual's ability to upregulate VEGF expression could be tested. Several million years later, nature may begin to favor survival of those best equipped to adapt to the threats posed by tissue ischemia. The genetic endowment permitting certain individuals to upregulate cytokine expression in a fashion that is optimally suited to revascularize ischemic tissues may constitute a distinct survival advantage. In the shorter term, the ability to recognize critical genetic features of such individuals may permit us to identify people who are least capable of mounting a satisfactory response and to develop appropriate therapeutic interventions to help them.

\section{Data supplement}

Supplemental references for this article can be found on the JCI web site. http://www.jci.org/

\footnotetext{
1. Avivi, A., Resnick, M.B., Nevo, E., Joel, A., and Levy, A.P. 1999. Adaptive hypoxic tolerance in the subterranean mole rat Spalax ehrenbergi: the role of vascular endothelial growth factor. FEBS Lett. 452:133-140.

2. Vale, P.R., et al. 2000. Left ventricular electromechanical mapping to assess efficacy of $\mathrm{phVEGF}_{165}$ gene transfer for therapeutic angiogenesis in chronic myocardial ischemia. Circulation. 102:965-974

3. Couffinhal, T., et al. 1998. A mouse model of angiogenesis. Am. J. Pathol. 152:1667-1679.

4. Li, J., et al. 1996. VEGF, flk-1, and $f l t-1$ expression in a rat myocardial infarction model of angiogenesis. Am. J. Physiol. 270:H1803-H1811.

5. Lee, S.H., et al. 2000. Early expression of angiogenesis factors in acute myocardial ischemia and infarction. N. Engl. J. Med. 342:626-633.

6. Ikeda, H.Y., et al. 2000. Expression of vascular endothelial growth factor in patients with acute myocardial infarction. J. Am. Coll. Cardiol. 35:968-973

7. D'Arcangelo, D., et al. 2000. Acidosis inhibits endothelial cell apoptosis and function and induces basic fibroblast growth factor expression. Circ. Res. 86:312-318.

8. Forsythe, J.A., et al. 1996. Activation of vascular endothelial growth factor gene transcription by hypoxia-inducible factor 1. Mol. Cell. Biol.
} 
16:4604-4613.

9. Shima, D.T., Deutsch, U., and D'Amore, P.A. 1995. Hypoxic induction of vascular endothelial growth factor (VEGF) in human epithelial cells is mediated by increases in mRNA stability. FEBS Lett. 370:203-208.

10. Stein, I., Neeman, M., Shweiki, D., Itin, A., and Keshet, E. 1995. Stabilization of vascular endothelial growth factor mRNA by hypoxia and hypoglycemia and coregulation with other ischemia-induced genes. Mol. Cell. Biol. 15:5363-5368.

11. Levy, A.P., et al. 1995. Regulation of vascular endothelial growth factor in cardiac myocytes. Circ. Res. 76:758-766.

12. Couffinhal, T., et al. 1999. Impaired collateral vessel development associated with reduced expression of vascular endothelial growth factor in ApoE-/- mice. Circulation. 99:3188-3198.

13. Arras, M., et al. 1998. Monocyte activation in angiogenesis and collateral growth in the rabbit hindlimb. J. Clin. Invest. 101:40-50.

14. Freeman, M.R., et al. 1995. Peripheral blood T lymphocytes and lymphocytes infiltrating human cancers express vascular endothelial growth factor: a potential role for $\mathrm{T}$ cells in angiogenesis. Cancer Res. 55:4140-4145.

15. Kalka, C., et al. 2000. Transplantation of ex vivo expanded endothelia progenitor cells for therapeutic neovascularization. Proc. Natl. Acad. Sci. USA. 97:3422-3427.

16. Brogi, E., et al. 1996. Hypoxia-induced paracrine regulation of VEGF receptor expression. J. Clin. Invest. 97:469-476.

17. Murohara, T., et al. 1998. Nitric oxide synthase modulates angiogenesis in response to tissue ischemia. J. Clin. Invest. 101:2567-2578.

18. Ziche, M., et al. 1997. Nitric oxide synthase lies downstream from vascular endothelial growth factor-induced but not fibroblast growth factor-induced angiogenesis. J. Clin. Invest. 99:2625-2634.

19. Tsurumi, Y., et al. 1997. Reciprocal relationship between VEGF and NO in the regulation of endothelial integrity. Nat. Med. 3:879-886.

20. Drake, C.J., and Little, C.D. 1995. Exogenous vascular endothelial growth factor induces malformed and hyperfused vessels during embryonic neovascularization. Proc. Natl. Acad. Sci. USA. 92:7657-7661.

21. White, F.C., Carroll, S.M., Magnet, A., and Bloor, C.M. 1992. Coronary collateral development in swine after coronary artery occlusion. Circ. Res. 71:1490-1500.

22. Takeshita, S., et al. 1997. Use of synchrotron radiation microangiography to assess development of small collateral arteries in a rat model of hindlimb ischemia. Circulation. 95:805-808.

23. Mandriota, S.J., and Pepper, M.S. 1998. Regulation of angiopoietin-2 mRNA levels in bovine microvascular endothelial cells by cytokines and hypoxia. Circ. Res. 83:852-859.

24. Brogi, E., Wu, T., Namiki, A., and Isner, J.M. 1994. Indirect angiogenic cytokines upregulate VEGF and bFGF gene expression in vascular smooth muscle cells, while hypoxia upregulates VEGF expression only. Circulation. 90:649-652.

25. Breier, G., Albrecht, U., Sterrer, S., and Risau, W. 1992. Expression of vascular endothelial growth factor during embryonic angiogenesis and endothelial cell differentiation. Development. 114:521-532.

26. Shi, Q., et al. 1998. Evidence for circulating bone marrow-derived endothelial cells. Blood. 92:362-367.

27. Takahashi, T., et al. 1999. Ischemia- and cytokine-induced mobilization of bone marrow-derived endothelial progenitor cells for neovascularization. Nat. Med. 5:434-438.

28. Rivard, A., et al. 1999. Age-dependent impairment of angiogenesis. Circulation. 99:111-120.

29. Rivard, A., et al. 2000. Age-dependent defect in VEGF expression is associated with reduced HIF-1 activity. J. Biol. Chem. In press.

30. Frenkel-Denkberg, G., Gershon, D., and Levy, A.P. 1999. The function of hypoxia-inducible factor 1 (HIF-1) is impaired in senescent mice. FEBS Lett. 462:341-344.

31. Kalka, C., et al. 2000. VEGF 165 gene transfer augments circulating endothelial progenitor cells in human subjects. Circ. Res. 86:1198-1202.

32. Van Belle, E., et al. 1997. Hypercholesterolemia attenuates angiogenesis but does not preclude augmentation by angiogenic cytokines. Circulation. 96:2667-2674.

33. Vincent, K.A., et al. 2000. Angiogenesis is induced in a rabbit model of hindlimb ischemia by naked DNA encoding a HIF-1 $\alpha / V P 16$ hybrid transcription factor. Circulation. In press.

34. Rivard, A., et al. 1999. Rescue of diabetes related impairment of angiogenesis by intramuscular gene therapy with adeno-VEGF. Am. J. Pathol. 154:355-364.

35. Abaci, A., et al. 1999. Effect of diabetes mellitus on formation of coronary collateral vessels. Circulation. 99:2239-2242.

36. Rohan, R.M., Fernandez, A., Udagawa, T., Yuan, J., and D'Amato, R.J. 2000. Genetic heterogeneity of angiogenesis in mice. FASEB J. 14:871-876.

37. Schultz, A., et al. 1999. Interindividual heterogeneity in the hypoxic regulation of VEGF: significance for the development of the coronary artery collateral circulation. Circulation. 100:547-552.

38. Carmeliet, P., et al. 1999. Impaired myocardial angiogenesis and ischemic cardiomyopathy in mice lacking the vascular endothelial growth factor isoforms VEGF ${ }_{188}$. Nat. Med. 5:495-502.

39. Marti, H., and Risau, W. 1998. Systemic hypoxia changes the organ specific distribution of vascular endothelial growth factor and its receptors. Proc. Natl. Acad. Sci. USA. 95:15809-15814.

40. Peterson, A., et al. 2000. Heterogeneity of the angiogenic factor/vascular endothelial growth factor. Lab. Invest. 80:99-115.

41. Hanahan, D., and Folkman, J. 1996. Patterns and emerging mechanisms of the angiogenic switch during tumorigenesis. Cell. 86:353-364. 